\title{
Simulations of High Harmonic Fast Wave Heating on the C-2U Advanced Beam-Driven Field-Reversed Configuration Device
}

\author{
Xiaokang Yang ${ }^{1, *}$, Yuri Petrov $^{2}$, Francesco Ceccherini ${ }^{1}$, Alf Koehn $^{3}$, Laura Galeotti ${ }^{1}$, Sean Dettrick ${ }^{1}$, Michl Binderbauer ${ }^{1}$, \\ and the TAE Team \\ ${ }^{1}$ Tri Alpha Energy, Inc., P.O. Box 7010, Rancho Santa Margarita, CA 92688, USA \\ ${ }^{2}$ CompX, P.O. Box 2672, Del Mar, CA 92014, USA \\ ${ }^{3}$ IGVP, University of Stuttgart, Pfaffenwaldring 31, 70569 Stuttgart, Germany
}

\begin{abstract}
Numerous efforts have been made at Tri-Alpha Energy (TAE) to theoretically explore the physics of microwave electron heating in field-reversed configuration (FRC) plasmas. For the fixed 2D profiles of plasma density and temperature for both electrons and thermal ions and equilibrium field of the C-2U machine, simulations with GENRAY-C ray-tracing code have been conducted for the ratios of $\omega / \omega_{c i[D]}$ in the range of $6-20$. Launch angles and antenna radial and axial positions have been optimized in order to simultaneously achieve good wave penetration into the core of FRC plasmas and efficient power damping on electrons. It is found that in an optimal regime, single pass absorption efficiency is $100 \%$ and most of the power is deposited inside the separatrix of FRC plasmas, with power damping efficiency of about $72 \%$ on electrons and less than $19 \%$ on ions. Calculations have clearly demonstrated that substantial power absorption on electrons is mainly attributed to high beta enhancement of magnetic pumping; complete power damping occurs before Landau damping has a significant effect on power absorption.
\end{abstract}

\section{Introduction}

The C-2U advanced beam-driven FRC device [1] is a simple compact toroid magnetic confinement system, that is, one without toroidal coils linking the plasma, and thus with predominantly poloidal fields. The attractions of such a configuration for a potential fusion reactor are its very high $\left\langle\beta_{e}\right\rangle$ (near unity) thus allowing for efficient use of magnetic hardware, simple and linear geometry for ease of construction and maintenance, as well as a natural, unrestricted divertor configuration for facilitating energy extraction and fusion ash removal [2].

However, the unique characteristics of C-2U FRC plasmas, for example, the plasma being unusually overdense $\left(\omega_{p e}>30 \omega_{c e}\right.$ inside the separatrix) and the magnetic field dropping quickly to zero in the plasma core, make it extremely challenging to heat electrons in the core of FRC plasmas. Conventional electron heating scenarios such as electron cyclotron resonant frequency (or its second or third harmonics) heating which is widely utilized in tokamaks, stellarators, and mirror machines, cannot be adapted to FRC plasmas due to the issue of poor wave accessibility into the plasma core. Other electron heating scenarios, such as electron Bernstein waves, upper-hybrid resonant waves, and whistler waves, encounter similar problem or have low heating efficiency when they are applied to FRC plasmas $[3,4]$. Fortunately, our recent survey indicated that the conflict between good wave accessibility and efficient power damping on electrons may be solved by using high harmonic fast wave (HHFW) heating, which has been successfully adapted to high beta, overdense spherical tokamak (ST) plasmas such as NSTX for the experiments of core electron heating and off-axis current drive [5 - 7].

It is well known that in the regime of fast wave direct electron heating, there are two collisionless damping mechanisms: Landau damping (LD), where the force acting on electrons is $\boldsymbol{F}_{\mathrm{LD}}=e \boldsymbol{E}_{/ /}$; and transit-time magnetic pumping (TTMP or MP), in which the force is $\boldsymbol{F}_{\mathrm{MP}}=-\nabla_{/(}\left(\mu B_{/ /}\right)$. Here $e$ and $\mu$ are electron's charge and magnetic moment, and $\boldsymbol{E}_{/ /}$and $B_{/ /}$are the parallel components of the fast wave electric and magnetic field, respectively. Conventional fast wave electron heating in tokamak plasmas requires wave parallel phase velocity $V_{\mathrm{ph} / /} \equiv \omega / k_{/ /} \approx V_{\mathrm{Te}}$ (electron thermal velocity) for any significant absorption via a dominated LD; MP makes no significant contribution to electron damping and often it can be neglected. Moreover, the absorption of fast wave in tokamak plasmas is weak and therefore it is usually required to have a strong electron preheating by microwaves at electron cyclotron resonant frequency in order to enhance multiple-pass power absorption. However, in high beta, ST plasmas like NSTX, it was found that the MP significantly increases power absorption on electrons over the electron LD alone, and it becomes substantially large at a higher range of phase velocity, $\omega / k_{/ /} \leq 2.5 V_{\mathrm{Te}}$ [5]. The combination of MP and LD can lead to $100 \%$ single pass absorption.

Corresponding author: xyang@trialphaenergy.com 


\section{Parameter settings}

Ray-tracing code Genray-C is a special version of Genray [8] adapted for plasmas like FRC or mirror machine where toroidal magnetic field can be absent. The C-2U machine configuration can be found in [1]; pure deuterium gas is routinely used in experiments. In our simulations, 5 rays are launched from one point but with different angles, each ray carrying $200 \mathrm{~kW}$ power. In this report, the launcher's $\mathrm{z}$-axial position is fixed at the midplane $(z=0)$, which is a preferable position for HHFW antenna-plasma coupling and wave penetration; moreover, launching HHFW at the midplane can make antenna physics and engineering design easier. The 2D equilibrium field profile reconstructed from experimental measurements is utilized; Fig. 1 shows its radial profile at the midplane. For feasibility study, the 2D model density profiles are used, matching those from C-2U experimental measurements. Both electrons and ions (D) have the same peak density of $2.4 \times 10^{19} \mathrm{~m}^{-3}$ at the field null point and the same density of $1.5 \times 10^{19} \mathrm{~m}^{-3}$ at the separatrix. In this case, the plasma has an unusually large (comparing to tokamaks) dielectric constant $\varepsilon\left(\equiv \omega_{\mathrm{pe}}{ }^{2} / \omega_{\mathrm{ce}}{ }^{2}>4000\right.$ inside the separatrix layer $)$. The $T_{\mathrm{e}}$ and $T_{\mathrm{i}}$ (thermal $\mathrm{D}$ ions) in the plasma core are $150 \mathrm{eV}$ and $800 \mathrm{eV}$, respectively. For simplification the data (density and temperature) on fast ions generated by neutral-beam injection is not included in present calculations. The simulations with fast ions will be performed in future work.

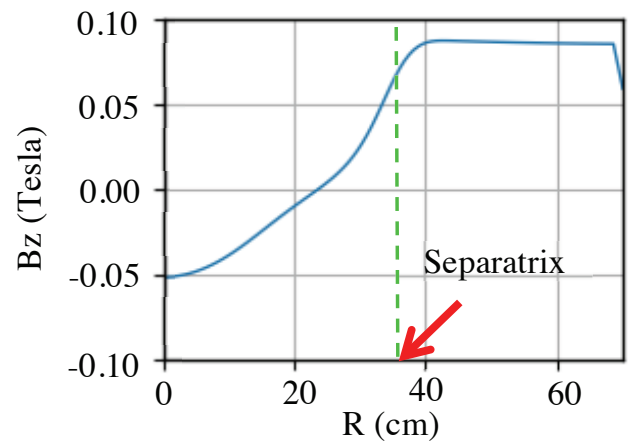

Fig. 1. Radial profile of C-2U equilibrium field $B_{z}$ at midplane.

\section{Simulation results}

Extensive simulations have been performed in order to find optimal regimes, for which the HHFWs have both good penetration and favourable power damping partition between electrons and ions. The parameters that have been scanned in the process of optimization include frequencies (thus the ratio of $\omega / \omega_{c i[D]}$ at rays' starting point since equilibrium field profile is fixed), antenna radial positions (the distance between antenna surface and plasma edge), and spectrum of parallel refractive index $n_{/ /}$at the antenna position (launch angles). Those parameters can have significant effects on wave coupling, propagation, and penetration, as well as power damping partition between electrons and ions.
As an example of optimal regimes, Fig. 2 shows HHFW propagation trajectory in C-2U FRC plasmas. The HHFW frequency is at $7 \mathrm{MHz}$, and the initial $n_{/ /}$is in a narrow range between 5 and 7 for the five rays. The background contours show the $2 \mathrm{D}$ profile of electron density, which is peaked at magnetic field null or local minimum of $|B|$. Rays launched at the possible antenna position near the wall $(\mathrm{z}=0, \mathrm{r}=55 \mathrm{~cm})$ can penetrate through the separatrix layer and propagate into the FRC plasma core. Figure 3 shows the change of power in each ray channel with the distance of wave propagation. The total distance of the rays' propagation is about $200 \mathrm{~cm}$, but most of their power is deposited by non-collisional damping in the area between $40 \mathrm{~cm}$ to $100 \mathrm{~cm}$ (the region marked with shadow); even though rays can continue propagating another $100 \mathrm{~cm}$ into the plasma core, the remaining power is very small.

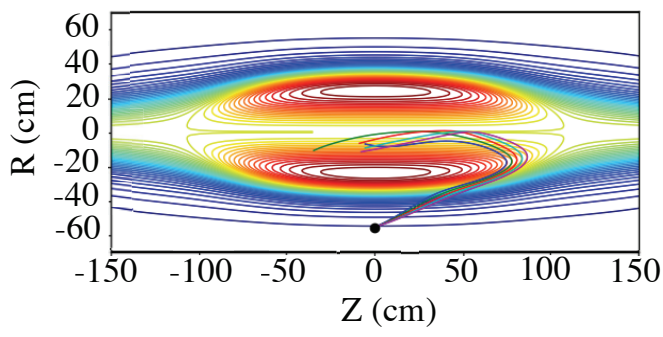

$\begin{array}{llllll}5 \mathrm{e} 11 & 5.5 \mathrm{e} 12 & 1.0 \mathrm{e} 13 & 1.6 \mathrm{e} 13 & 2.0 \mathrm{e} 13 & \left(\mathrm{~cm}^{-3}\right)\end{array}$

Fig. 2. The propogation trajectory of $\mathrm{HHFW}(7 \mathrm{MHz})$ in $\mathrm{C}-2 \mathrm{U}$ plasmas. Background contours show electron density profile.

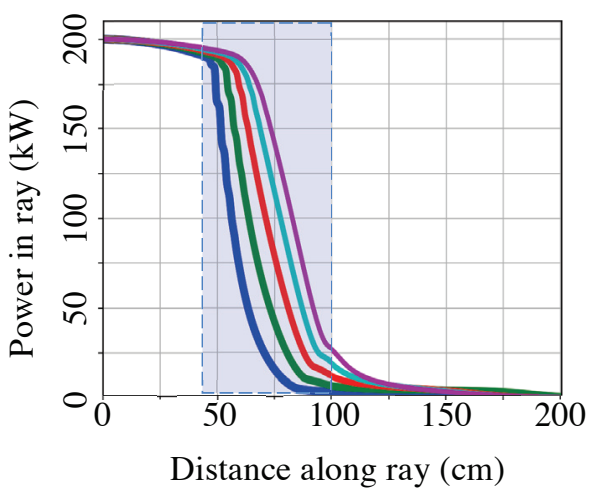

Fig. 3. The HHFW (7 MHz) power in ray channel along the distance of wave propagation. The initial $n_{/ /}=5$ for blue ray and $n_{/ /}=7$ for purple ray.

The radial profiles of power density for total power absorption, power damping on electrons and ions, and collisional power damping, are shown in Figs. 4(a)-4(d), respectively. The position of rho $=1$ corresponds to the location of separatrix layer of FRC plasma, where rho is defined through the square root of poloidal flux. In this report, the area of rho $<1$ (with closed field lines) defines "plasma core" while the area of rho $>1$ outlines the region of scrape-off layer (SOL). As shown clearly in Fig. 4(a), single pass absorption efficiency of HHFW power is $100 \%$ (total launched power is $1000 \mathrm{~kW}$ ), and 
most power (around 60\%) is damped inside the separatrix. Also, Figs. 4(b) and 4(c) indicate that the power partition is $72 \%$ on electrons and about $19 \%$ on ions. The remaining power (about 9\%) is damped through collisions (Fig. 4(d)) due to a relatively low electron temperature at plasma edge. The "sawteeth"like features seen in profiles in Figs. 4(a) and 4(c) represent ion cyclotron harmonic resonance damping.

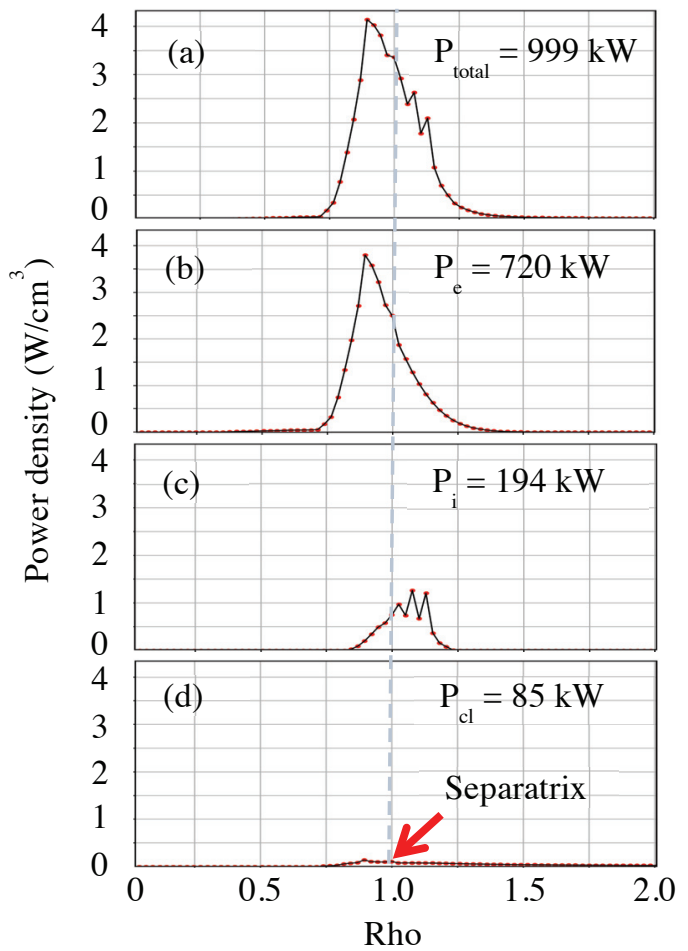

Fig. 4. Radial profiles of power density for (a) total absorption, (b) damping on electrons, (c) damping on ions, and (d) collisional damping.

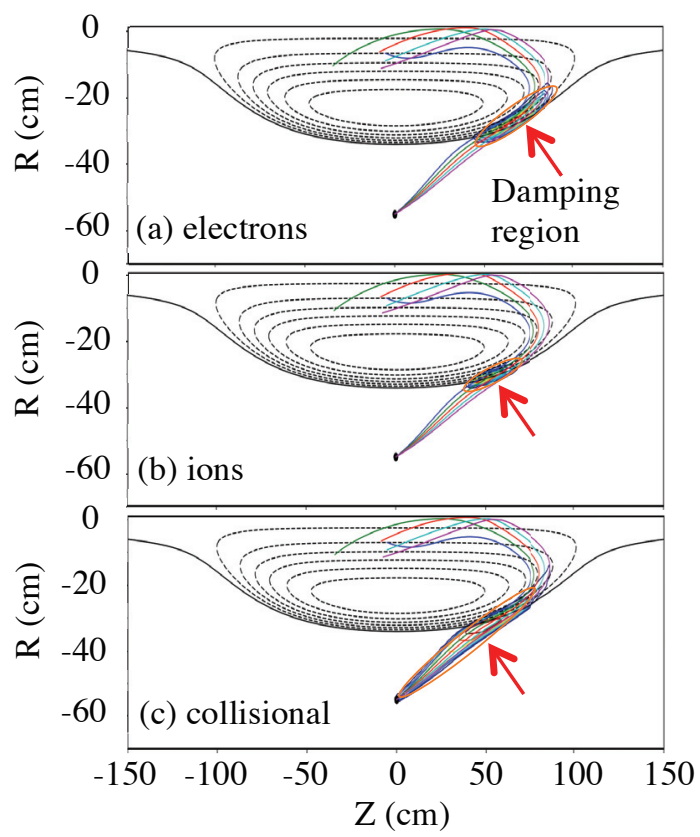

Fig. 5. The 2D profiles of power density for (a) damping on electrons, (b) damping on ions, and (c) collisional damping. Background contours show magnetic flux 2D profile.
The $2 \mathrm{D}$ power density profiles of damping on electrons, ions, and collisional damping are plotted in Fig. 5 with the marked area showing power damping region. As can be seen clearly in Fig. 5, the power damping regions for electrons and ions are overlapped, thus there is a competition of power partition between electrons and ions when HHFW propagates into this overlapped area.

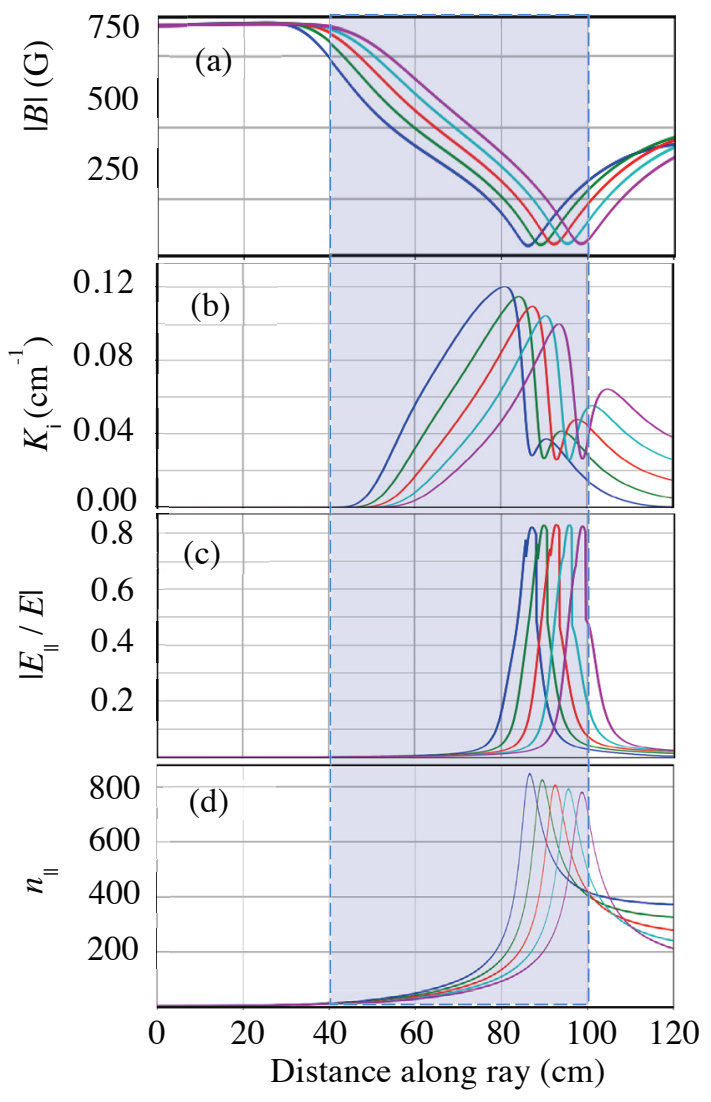

Fig. 6. Changes of (a) local $|B(\mathrm{r}, \mathrm{z})|$, (b) imaginary part of perpendicular wave number $k_{\mathrm{i}}$, (c) ratio of $\left|E_{/ /} / E\right|$, and (d) parallel refractive index $n_{/ /}$, with the distance along wave propagation.

Figure 6 can help to better understand the damping mechanisms of HHFW in FRC plasmas, which shows the changes of local magnetic field $\left(|B(\mathrm{r}, \mathrm{z})|=\left(B_{\mathrm{r}}^{2}\right.\right.$ $\left.+B_{\mathrm{z}}{ }^{2}\right)^{1 / 2}$ ), imaginary part of perpendicular wave number $k_{\mathrm{i}}$, the normalized parallel component of wave electric field $\left|E_{/ /} / E\right|$, and parallel refractive index $\mathrm{n}_{/ /}$, with the distance along wave propagation. The value of $k_{\mathrm{i}}$ in Fig. 6(b) describes power damping on electrons by both MP and LD; although we are presently unable to separate the contribution of MP and LD, we can use Fig. 6(c) to conclude where LD can have an effect of power damping on electrons because $\boldsymbol{F}_{\mathrm{LD}}=e \boldsymbol{E}_{/ /}$. Clearly, if $\left|E_{/ /} / E\right|$ is small, there can be no pronounced LD. Meanwhile, according to Ref. [5], MP is strongly related to the local magnetic field, and it is found to scale as $\propto$ $B^{-3}$.

As can be seen in Figs. 6(b) and 6(c), there is no collisionless damping on electrons at a distance of wave propagation from 0 to $40 \mathrm{~cm}$ because the equilibrium magnetic field remains large and at the same time the value of $\left|E_{/ /} / E\right|$ is nearly zero. A significant power 
damping occurs in the area between $40 \mathrm{~cm}$ and $100 \mathrm{~cm}$ (also see the marked area in Fig. 3) due to a rapid drop of magnetic field (Fig. 6(a)), which leads to fast increase of $k_{\mathrm{i}}$ (Fig. 6(b)). For the ray with blue color $\left(n_{/ /}=5\right)$ in Fig. 6(c), the value of $\left|E_{/ /} / E\right|$ is almost zero from $40 \mathrm{~cm}$ to $70 \mathrm{~cm}$; this value is still less than 0.2 even when $k_{\mathrm{i}}$ reaches a maximum value at the distance of $82 \mathrm{~cm}$, where $\omega / k_{/ /} \leq 0.5 V_{\mathrm{Te}}$ (this condition is different from either fast wave in tokamak plasmas or HHFW in ST plasmas). The value of $k_{\mathrm{i}}$ drops to less than $1 / 3$ of its maximum value when $\left|E_{/ /} / E\right|$ reaches a maximum at 87 $\mathrm{cm}$, where $|B(\mathrm{r}, \mathrm{z})|$ has a minimum and $n_{/ /}$has a maximum. Therefore, conclusion can be made that the quick increase of $k_{\mathrm{i}}$ is mainly attributed to high beta enhancement of MP, and power damping on electrons is almost completed before LD has a significant effect on power absorption.

\section{Discussions}

Through extensive calculations, it is found that efficient HHFW electron heating in $\mathrm{C}-2 \mathrm{U}$ plasmas is more difficult to realize than the case of HHFW heating in ST plasmas. The unique magnetic configuration of FRC plasmas can cause the conflict between good wave penetration and favorable power partition between electrons and ions. In C-2U FRC plasmas, the parameters such as the ratio of $\omega / \omega_{c i[D]}$, the initial value of $n_{/ /}$, the distance between antenna and plasma edge, and the launcher position along $\mathrm{z}$ axis, have significant impact not only on wave coupling and penetration, but also on power partition between electrons and ions. While in spherical tokamaks like NSTX, there is no issue of wave penetration into plasma core, thus one can only focus on how to avoid a parasitic ion heating or mitigate hot ion damping.

In $\mathrm{C}-2 \mathrm{U}$ plasmas, on the one hand, in order to mitigate the competition from ion heating, one can maximize power damping on electrons by increasing (1) the ratio of $\omega / \omega_{c i[D]}$, or (2) the initial value of $n_{/ /}$, or (3) the distance between antenna and plasma edge. However, the changes of those parameters may shift the area of power damping from inside the separatrix layer into the SOL. As a result, electrons at open field lines will be heated and therefore fast wave power will eventually be lost (at end divertors). Those results are somehow similar to the regime of Whistler wave heating.

On the other hand, in order to enhance HHFW penetration into the core of FRC plasmas, one can reduce (1) the ratio of $\omega / \omega_{c i[D]}$, or (2) the initial value of $n_{/ /}$, or (3) the distance between antenna and plasma edge. However, although single pass absorption efficiency is still $100 \%$ in those cases, power absorption by electrons decreases substantially and most of the HHFW power is deposited to the ions through ion (D) cyclotron harmonic resonance absorption. By comparing with the case shown in Fig. 2 ( $\mathrm{f}=7 \mathrm{MHz}$ and $n_{/ /}=5-7$ ), one can find that the case shown in Fig. $7\left(\mathrm{f}=5 \mathrm{MHz}\right.$ and $n_{/ /}=3-5$ ) has a better wave penetration (getting more closely to the region of magnetic field null) and stronger power absorption (shorter distance along wave propagation). Calculations indicate that for the case plotted in Fig. 7, power absorption partition is around $15 \%$ on electrons and $75 \%$ on ions, and the remainder of $10 \%$ power is deposited through collisional damping.

In summary, our simulation results have demonstrated for the first time that the HHFW heating scenario can offer a promising path to efficient heating of electrons in the core of FRC plasmas. In a wide range of plasma parameters, single pass power absorption efficiency is $100 \%$; power damping on electrons increases quickly with the fast drop of magnetic field strength in the core of FRC plasma, and it reaches a maximum value before LD has any significant effect on power absorption. It is obvious that MP is the dominated power absorption mechanism for HHFW electron heating in FRC plasmas.

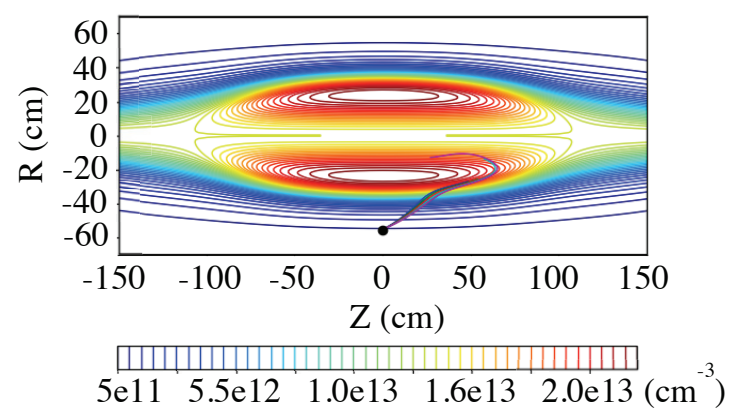

Fig. 7. The propagation trajectory of $\mathrm{HHFW}(5 \mathrm{MHz})$ in $\mathrm{C}-2 \mathrm{U}$ plasmas. Background contours show electron density profile.

We would like to acknowledge the support and contributions from the rest of the TAE team and collaborators. A debt of gratitude is also due to our shareholders for their continuous support.

\section{References}

1. M. Binderbauer, et al., Phys. Plasmas, 22, 056110 (2015).

2. M. Tuszewski, Nucl. Fusion, 28, 2033 (1988).

3. X. Yang, et al., 21st Topical Conf. On RF Power in Plasmas, AIP Conference Proceedings 1689, 090081 (2015).

4. X. Yang, et al., Bull. Am. Phys. Soc. 58, PP10.00013 (2016).

5. M. Ono, Phys. Plasmas, 11, 4075(1995).

6. J. Hosea, et al., Phys. Plasmas, 15, 056104 (2008)

7. G. Taylor, et al., Phys. Plasmas, 17, 056114 (2010)

8. A. Smirnov and R. Harvey, CompX report, CompX2000-01 (2001). 